\title{
Overexpression of MYC and BCL2 Predicts Poor Prognosis in Patients with Extranodal NK/T-cell Lymphoma, Nasal Type
}

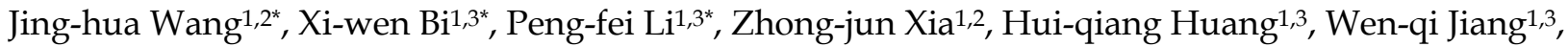 \\ Yu-jing Zhang, ${ }^{1,4}$, Liang Wang $1,2 \bowtie$ \\ 1. State Key Laboratory of Oncology in South China; Collaborative Innovation Center for Cancer Medicine, Guangzhou, Guangdong, 510060, People's \\ Republic of China \\ 2. Department of Hematologic Oncology, Sun Yat-sen University Cancer Center, Guangzhou, Guangdong, 510060, People's Republic of China \\ 3. Department of Medical Oncology, Sun Yat-sen University Cancer Center, Guangzhou, Guangdong, 510060, People's Republic of China \\ 4. Department of Radiation Oncology, Sun Yat-sen University Cancer Center, Guangzhou, Guangdong, 510060, People's Republic of China \\ *The first three authors contributed equally to this work
}

$\triangle$ Corresponding author: Liang Wang, M.D., Department of Hematologic Oncology, State Key Laboratory of Oncology in South China/Cancer Center, Collaborative Innovation Center for Cancer Medicine, Sun Yat-sen University, Guangzhou, Guangdong 510060, P.R. China. E-mail: wangliang@sysucc.org.cn; Tel..+86 20 87342439; Fax: +86 2087342439.

(c) Ivyspring International Publisher. This is an open access article distributed under the terms of the Creative Commons Attribution (CC BY-NC) license (https://creativecommons.org/licenses/by-nc/4.0/). See http://ivyspring.com/terms for full terms and conditions.

Received: 2016.09.29; Accepted: 2016.11.14; Published: 2017.02.25

\begin{abstract}
Background: Recently double-hit lymphoma or double protein expressor lymphoma has been identified as a distinct group of diffuse large B cell lymphoma with poor prognosis. However, the expression status, clinical and prognostic effect of combined overexpression of MYC and BCL2 in extranodal NK/T-cell lymphoma, nasal type (ENKTL) are not known.

Materials and methods: Paraffin-embedded lymphoma samples from 53 patients with newly diagnosed ENKTL were studied using immunohistochemistry for MYC and BCL2, and fluorescent in situ hybridization (FISH) for MYC and BCL2 were done on 5 tissue sections with highest percentages of both MYC and BCL2 positive lymphoma cells.

Results: The median percentage of MYC-positive lymphoma cells and BCL2-positive lymphoma cells were $20 \%$ (range, $5 \%-45 \%$ ) and $70 \%$ (10\%-95\%), respectively. Using median scores as cutoffs, we assigned each patient an IHC double-hit score (DHS) that ranged from 0 to 2. Using this DHS, 15 patients $(28.3 \%)$ had a DHS of 0,24 patients $(45.3 \%)$ had a DHS of 1 , and the remaining 14 patients (26.4\%) had a DHS of 2. FISH analysis was performed on 5 tissue sections with DHS of 2, and none of them had MYC or BCL2 rearrangement. The DHS was not associated with patients' age, gender, disease stage, LDH level, B symptoms, performance status, or local tumor invasiveness. However, patients with tumor localized in extranasal sites seemed to have higher expression of BCL2 and higher DHS than nasal lesions ( $p=0.014$ and 0.042 , respectively). In univariate survival analysis, either high expression of MYC or BCL2 was significantly correlated with inferior PFS and OS $(p<0.05)$. According to the DHS, patients with ENKTL could be divided into three significantly different risk groups for PFS and OS (3-year PFS rate for DHS of 0, 1, and 2 was $60 \%, 41 \%$, and $21 \%$, respectively, $\mathrm{p}=0.008 ; 3$-year OS rate for $\mathrm{DHS}$ of 0,1 , and 2 was $79 \%, 49 \%$, and $33 \%$, respectively, $p=0.015$ ). In multivariate survival analysis, it was found that DHS was an independent prognostic factor for both PFS and OS ( $\mathrm{p}=0.006$ and 0.011 , respectively).

Conclusions: Our study demonstrated that DHS can help identify patients with newly diagnosed ENKTL who are at a high risk for a poor clinical outcome, which needs to be validated in prospective clinical trials with patients treated uniformly.
\end{abstract}

Key words: extranodal NK/T-cell lymphoma, double-hit score, MYC, BCL-2, prognosis. 


\section{Introduction}

Extranodal NK/T-cell lymphoma, nasal type (ENKTL), a distinct type of non-Hodgkin lymphoma, is closely associated with Epstein-Barr virus infection, and relatively common in East Asia, Southeast Asia, and Central and South America[1,2]. Most patients of ENKTL have early stage disease at diagnosis, and thus radiotherapy (RT) with or without chemotherapy (CT) have been adopted as the primary treatment, leading to relatively favorable outcomes[3]. Previous studies have demonstrated poor efficacy with anthracycline-based CT (such as $\mathrm{CHOP}$ or $\mathrm{EPOCH}$ regimens) due to overexpression of multi-drug resistance (MDR) gene in ENKTL cells[4]. Recently, asparaginase-based CT regimens have been found to be highly efficacious in the treatment of ENKTL[5-7]. However, the prognosis of patients with advanced stage ENKTL is still very poor in spite of use of novel agents.

Previous studies have demonstrated that the international prognostic index (IPI) is not suitable for ENKTL because of imbalance of patient distribution[8,9]. Based on four parameters (B symptoms, Ann Arbor stage, LDH level, and regional lymph node involvement), a Korean Prognostic Index (KPI) is formed, which seems better than IPI[10]. Both IPI and KPI were developed on the basis of cohorts of patients who were primarily treated with cyclophosphamide, doxorubicin, vincristine, and prednisone (CHOP) or CHOP-like regimens[10,8]. With the advent of non-anthracycline-based CT, a new prognostic model (the prognostic index for natural killer cell lymphoma, PINK) is developed to predict outcomes in ENKTL[11]. However, all those prognostic models were based mainly on clinical characteristics, and cannot reflect the biology of ENKTL comprehensively.

In recent years, increasing studies have confirmed that translocation of the MYC gene, a gene that is involved in many cellular functions including proliferation, and BCL2, a central anti-apoptotic gene, are markers of poor prognosis in diffuse large $\mathrm{B}$ cell lymphoma (DLBCL)[12]. DLBCL with translocation of both MYC and BCL2, termed double-hit lymphoma (DHL), is featured by inferior response to standard therapy and unfavorable outcomes[13,14]. However, both the protein products, MYC and BCL2 can be overexpressed through other mechanisms without translocation of both genes[15]. Green et al has shown that immunohistochemical double-hit score (DHS, based on expression status of MYC and BCL2 protein) can predict strongly the outcomes of patients with DLBCL treated with rituximab plus CHOP (RCHOP) independent of gene translocations [15]. ENKTL, an EBV-associated lymphoma, is featured by overexpression of latent membrane protein-1 (LMP-1)[16], which has been demonstrated to be able to induce the expression of both MYC and BCL2. However, the expression status, clinical and prognostic effect of combined overexpression of MYC and BCL2 in ENKTL are not known. In this study, we investigated whether the immunohistochemical DHS can predict outcomes in patients with newly diagnosed ENKTL.

\section{Materials and Methods}

\section{Patients}

53 patients with newly diagnosed ENKTL from Sun Yat-sen University Cancer Center were enrolled in this study. All these patients had complete clinical documents, received treatments and pathology tissue sections were available. Approval to review, analyze, and publish the data in this study was given by the Sun Yat-sen University Cancer Center Research Ethics Board. Informed consent for the collection of medical information was obtained from all patients at their first visit.

\section{Treatments}

All 53 patients received chemotherapy as primary treatment (anthracycline-based regimens [8], $n=24$; asparaginase-based regimens [5], $n=29$ ). For early stage patients, consolidative involved-field RT (IFRT) was delivered using 6-MeV linear accelerator using conventional planning RT, 3-dimensional conformal treatment planning or intensity modulated RT (IMRT).

\section{Immunohistochemistry}

Slides of formalin-fixed, paraffin-embedded tissues were stained with an anti-MYC antibody (Abcam, Y69, ab32072) and anti-BCL2 antibody (Abcam, Bcl2/100, ab117115) using a standard streptavidin peroxidase technique and chromogen diaminobenzidine. The staining pattern for MYC protein was distinctly nuclear, whereas staining for BCL2 protein showed a well-defined cytoplasmic staining pattern. The percentage of positive expression lymphoma cells was recorded and used for analysis. To make the determination of expression status more objective, the scoring was performed independently by two different pathologists. The interobserver reproducibility was assessed using the difference between the scorings of each observer. The mean of the scorings from each reader was then calculated and used for the statistical analysis.

\section{Interphase FISH Analysis}

FISH analysis was performed on 5 tissue sections with highest percentages of both MYC and BCL2 
positive lymphoma cells using break apart FISH DNA probes for c-MYC/8q24 and BCL2/18q21 (probes Y5410, Y5407; Dako A/S), as previously described [15]. Slides were evaluated under a fluorescence microscope equipped with appropriate filter sets.

\section{Statistical analysis}

OS was calculated from the time of diagnosis until death from any cause or until the time of the last follow-up visit for the surviving patients. Progression-free survival (PFS) was defined as the interval from the time of diagnosis to the time of first documented disease progression or relapse or to the time of the last follow-up visit. The chi-square test was used to calculate statistical group comparisons of categorical variables. Survival analysis was performed using the Kaplan-Meier method, and comparisons were calculated using the log-rank test. Univariate and Multivariate analysis was used to estimate the prognostic impact of different variables in OS and PFS using the Cox regression model. $\mathrm{P}<0.05$ was considered statistically significant, and all $\mathrm{P}$ values correspond to two-sided significance tests. Statistical analyses were performed using SPSS 16.0 software.

\section{Results}

\section{Patient characteristics and treatments}

All patients' characteristics are shown in Table 1. The median age was 44 years old (range 10-73), and 6 patients $(11.3 \%)$ were older than 60 years old. 36 patients $(67.9 \%)$ had stage I-II disease, and serum LDH level was elevated in only $35.8 \%$ patients. $34.0 \%$ of patients presented with local tumor invasiveness, and the most frequently affected regions included paranasal sinus, skull base, and facial skin. In this study, all patients received chemotherapy as primary treatment, and $45.3 \%$ of patients had anthracycline-based CT regimens, and the remaining $54.7 \%$ of patients had asparaginase-based CT regimens. After induction chemotherapy, $52.8 \%$ of patients attained complete remission (CR), and the CR rate was significantly higher in patients treated with asparaginase-based CT regimens $(75.9 \%$ vs. $20.8 \%$, $\mathrm{p}<0.05)$.

\section{MYC and BCL2 expression status}

The median percentage of MYC-positive lymphoma cells and BCL2-positive lymphoma cells were $20 \%$ (range, 5\%-45\%) and 70\% (10\%-95\%), respectively. Using median scores as cutoffs, we assigned each patient an IHC double-hit score (DHS) that ranged from 0 to 2 (Fig. 1). Each of the two markers with percentage higher than the median value was given one point. Thus, patients with both MYC of more than $20 \%$ and BCL2 of more than $70 \%$ had a DHS of 2. Using this DHS, 15 patients (28.3\%) had a DHS of 0,24 patients $(45.3 \%)$ had a DHS of 1 , and the remaining 14 patients (26.4\%) had a DHS of 2 . FISH analysis was performed on 5 tissue sections with DHS of 2, and none of them had MYC or BCL2 rearrangement. As is shown in Table 1, the DHS was not associated with patients' age, gender, disease stage, LDH level, B symptoms, performance status, or local tumor invasiveness. However, patients with tumor localized in extranasal sites seemed to have higher expression of BCL2 and higher DHS than nasal lesions ( $\mathrm{p}=0.014$ and 0.042 , respectively).

Table 1. Clinical characteristics and MYC and BCL-2 expression.

\begin{tabular}{|c|c|c|c|c|c|c|c|c|c|c|}
\hline \multirow[t]{2}{*}{ Parameters } & & \multicolumn{3}{|c|}{ MYC (N=53) } & \multicolumn{3}{|c|}{ BCL-2 (N=53) } & \multicolumn{3}{|c|}{ DHS (N=53) } \\
\hline & & $>20 \%$ & $\leq 20 \%$ & P value & $>70 \%$ & $\leq 70 \%$ & $P$ value & $0-1$ & 2 & P value \\
\hline \multirow[t]{2}{*}{ Gender } & Male & 17 & 15 & 0.652 & 15 & 17 & 0.911 & 22 & 10 & 0.505 \\
\hline & Female & 9 & 12 & & 11 & 10 & & 17 & 4 & \\
\hline \multirow[t]{2}{*}{ Age } & $>=60$ & 4 & 2 & 0.420 & 3 & 3 & 1.000 & 3 & 3 & 0.323 \\
\hline & $<60$ & 22 & 25 & & 23 & 24 & & 36 & 11 & \\
\hline \multirow[t]{2}{*}{ Ann Arbor stage } & I-II & 15 & 21 & 0.203 & 16 & 20 & 0.495 & 29 & 7 & 0.109 \\
\hline & III-IV & 11 & 6 & & 10 & 7 & & 10 & 7 & \\
\hline \multirow[t]{2}{*}{ B symptoms } & Yes & 11 & 15 & 0.490 & 15 & 11 & 0.337 & 18 & 8 & 0.694 \\
\hline & No & 15 & 12 & & 11 & 16 & & 21 & 6 & \\
\hline \multirow[t]{2}{*}{$\mathrm{LDH}$} & Elevated & 10 & 9 & 0.918 & 13 & 6 & 0.069 & 12 & 7 & 0.336 \\
\hline & Normal & 16 & 18 & & 13 & 21 & & 27 & 7 & \\
\hline \multirow[t]{2}{*}{ ECOG PS } & $0-1$ & 15 & 21 & 0.203 & 16 & 20 & 0.495 & 29 & 7 & 0.109 \\
\hline & $>=2$ & 11 & 6 & & 10 & 7 & & 10 & 7 & \\
\hline \multirow[t]{2}{*}{ Primary tumor site } & Nasal cavity & 16 & 20 & 0.495 & 13 & 23 & 0.014 & 30 & 6 & 0.042 \\
\hline & Extra-nasal & 10 & 7 & & 13 & 4 & & 9 & 8 & \\
\hline \multirow[t]{2}{*}{ LTI } & Yes & 10 & 8 & 0.698 & 9 & 9 & 1.000 & 13 & 5 & 1.000 \\
\hline & No & 16 & 19 & & 17 & 18 & & 26 & 9 & \\
\hline \multirow{2}{*}{$\begin{array}{l}\text { Induction CT } \\
\text { regimen }\end{array}$} & ASP-based & 13 & 16 & 0.688 & 14 & 15 & 1.000 & 23 & 6 & 0.468 \\
\hline & Non-ASP-based & 13 & 11 & & 12 & 12 & & 16 & 8 & \\
\hline \multirow[t]{2}{*}{ CT Response } & $\mathrm{CR}$ & 10 & 17 & 0.131 & 11 & 16 & 0.337 & 23 & 4 & 0.101 \\
\hline & Non-CR & 16 & 10 & & 15 & 11 & & 16 & 10 & \\
\hline
\end{tabular}

Abbreviations: DHS, double hit score; LDH, lactate dehydrogenase; ECOG, Eastern Cooperative Oncology Group; PS, performance status; LTI, local tumor invasion; CT, chemotherapy; ASP, asparaginase; $\mathrm{CR}$, complete response. 


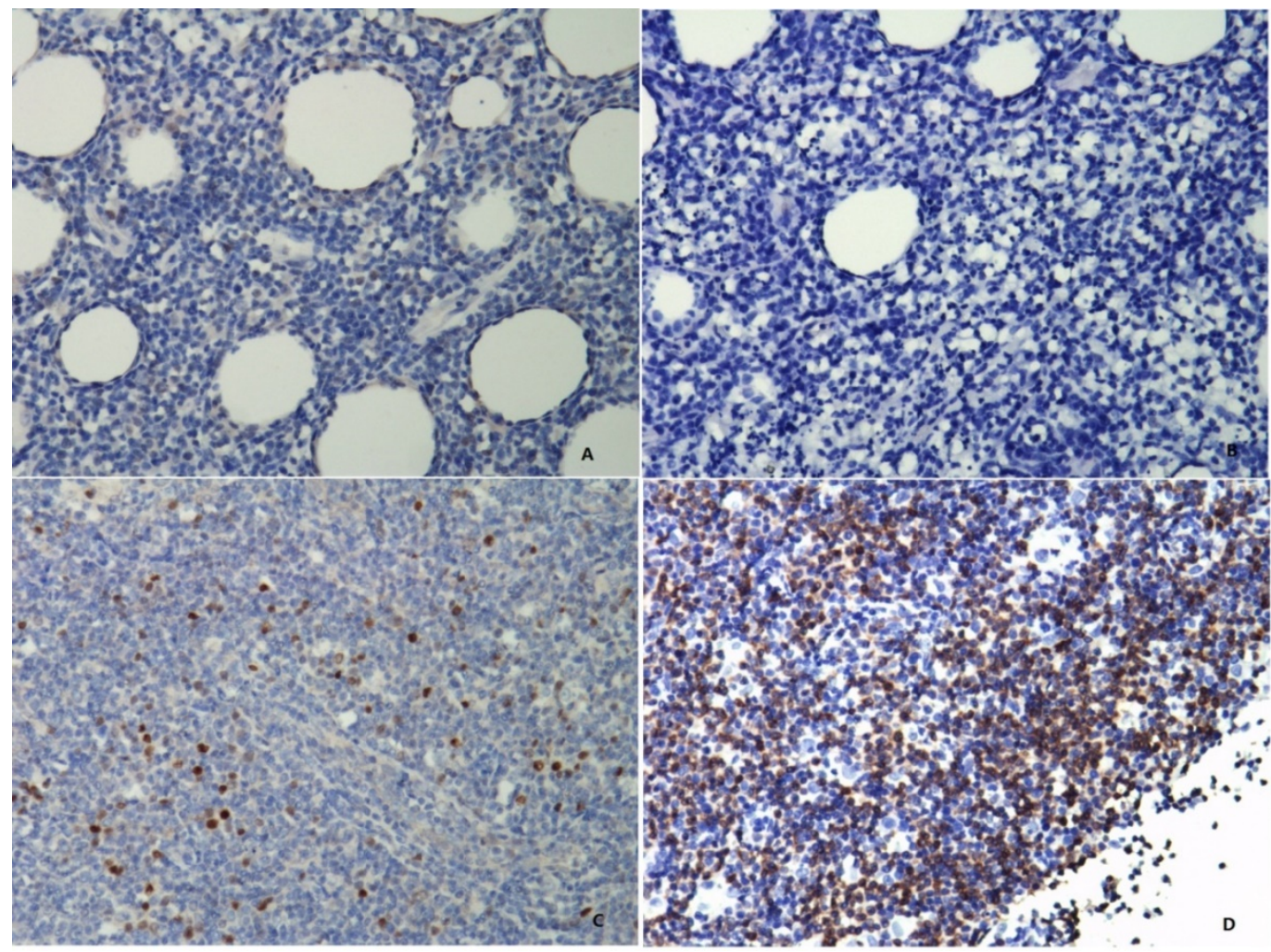

Figure 1. NK/T-cell lymphoma tissues stained for MYC and BCL2 protein (photographed at 200 magnifications). The MYC staining is mainly nuclear (A and C), whereas staining for BCL2 is predominantly a cytoplasmic pattern (B and D). Patients with (A) $\leq 20 \%$ MYC-positive lymphoma cells and (B) $\leq 70 \%$ BCL2-positive lymphoma cells are defined as double-hit score (DHS) of 0 . Patients with (C) $>20 \%$ MYC-positive lymphoma cells and (D) $>70 \%$ BCL2-positive lymphoma cells are defined as DHS of 2.

Table 2. Univariate and multivariate survival analysis.

\begin{tabular}{|c|c|c|c|c|c|c|}
\hline \multirow[t]{3}{*}{ Parameters } & \multicolumn{3}{|l|}{ PFS } & \multicolumn{3}{|l|}{ OS } \\
\hline & Univariate analysis & Multivariate analysis & & Univariate analysis & Multivariate analysis & \\
\hline & $\mathrm{p}$ value & $\mathrm{HR}(95 \% \mathrm{CI})$ & $\mathrm{p}$ value & $\mathrm{p}$ value & $\mathrm{HR}(95 \% \mathrm{CI})$ & $\mathrm{p}$ value \\
\hline Gender (F) & 0.744 & & & 0.766 & & \\
\hline Age $(>=60)$ & 0.492 & & & 0.934 & & \\
\hline Stage (III-IV) & 0.159 & & & 0.382 & & \\
\hline B symptoms (no) & 0.090 & & & 0.038 & $0.765(0.251-2.332)$ & 0.638 \\
\hline LDH (elevated) & 0.002 & $1.838(0.841-4.016)$ & 0.127 & 0.002 & $2.205(0.717-6.783)$ & 0.168 \\
\hline ECOG $(>1)$ & 0.001 & $1.081(0.402-2.913)$ & 0.877 & 0.001 & $2.506(1.065-5.897)$ & 0.035 \\
\hline LTI (yes) & 0.007 & 2.409 (1.094-5.307) & 0.029 & 0.002 & $2.949(1.229-7.081)$ & 0.015 \\
\hline $\begin{array}{l}\text { DHS } \\
\text { (2 vs.1 vs.0) }\end{array}$ & 0.008 & 1.986 (1.221-3.229) & 0.006 & 0.015 & $2.296(1.207-4.365)$ & 0.011 \\
\hline Induction CT (ASP) & 0.002 & $0.379(0.149-0.961)$ & 0.041 & 0.250 & & \\
\hline
\end{tabular}

Abbreviations: PFS, progression free survival; OS, overall survival; CI, confidence interval; HR, hazard ratio; F, female; LDH, lactate dehydrogenase; LTI, local tumor invasion; ECOG, Eastern Cooperative Oncology Group; CT, chemotherapy; ASP, asparaginase; DHS, double hit score.

\section{Survival analysis}

The median follow-up time for patients alive was 46 months (range, 14-106 months). The median PFS was 19.5 months, and the median OS was not reached, with the 3-year PFS and OS rate being $41 \%$ and $53 \%$, respectively. As is shown in Fig.2, either high expression of MYC or BCL2 was significantly correlated with inferior PFS and OS $(p<0.05)$. According to the DHS, patients with ENKTL could be divided into three significantly different risk groups for PFS and OS (3-year PFS rate for DHS of 0,1 , and 2 was $60 \%, 41 \%$, and $21 \%$, respectively, $\mathrm{p}=0.008$; 3-year
OS rate for DHS of 0,1 , and 2 was $79 \%, 49 \%$, and $33 \%$, respectively, $p=0.015)$. Patients treated with ASP-based induction therapy had significantly better PFS than those treated with non-ASP-based regimens $(\mathrm{P}=0.002$, Fig. $3 \mathrm{~A})$. However, there was no significant difference in OS between patients treated with ASP or non-ASP-based regimens ( $p=0.250$, Fig.3B). Patients who got $C R$ after induction therapy had significantly better PFS and OS than those without CR ( $<<0.01$ for both, Fig.3C and D). In multivariate survival analysis (Table 2), it was found that DHS was an independent prognostic factor for both PFS and OS $(p=0.006$ and 0.011 , respectively). 

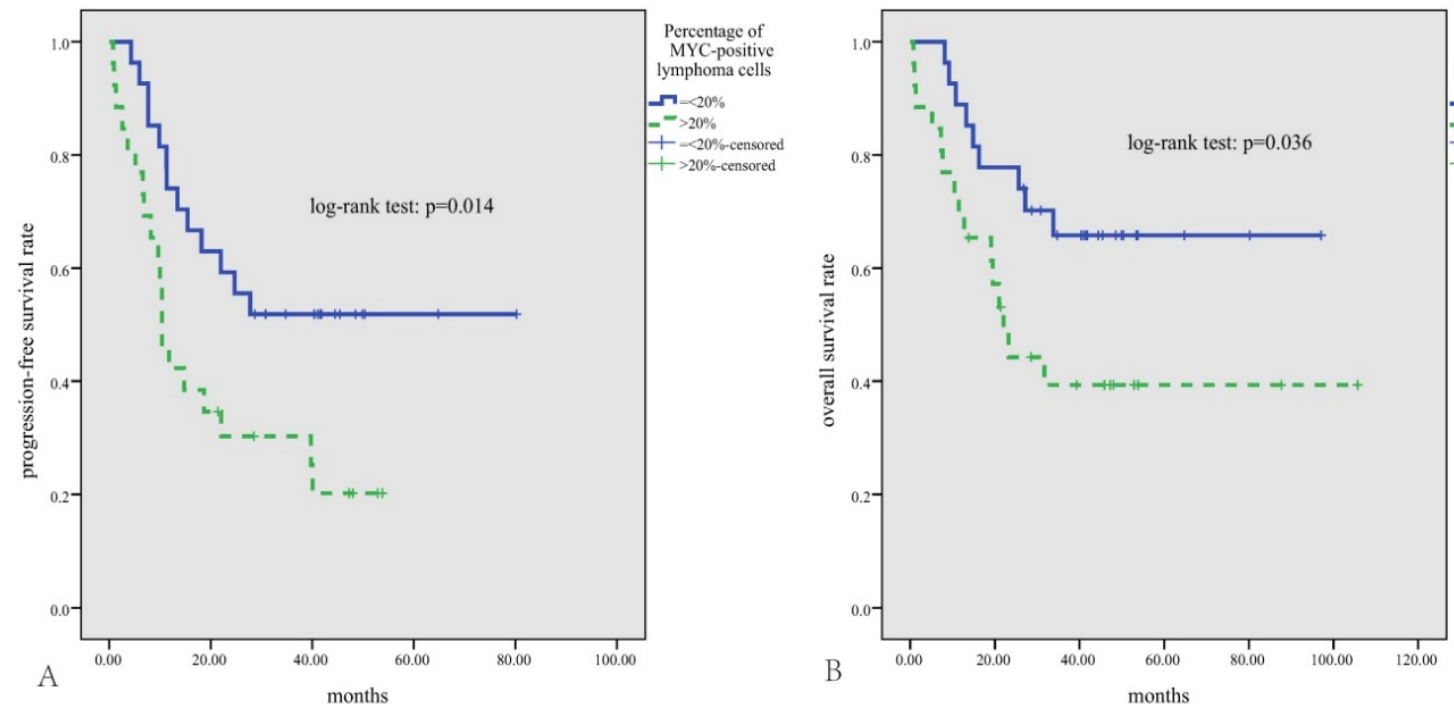

Percentage of MYC-positive lymphoma cells

I) $<20 \%$

$+<20 \%$-censored

$\rightarrow 20 \%$-censored
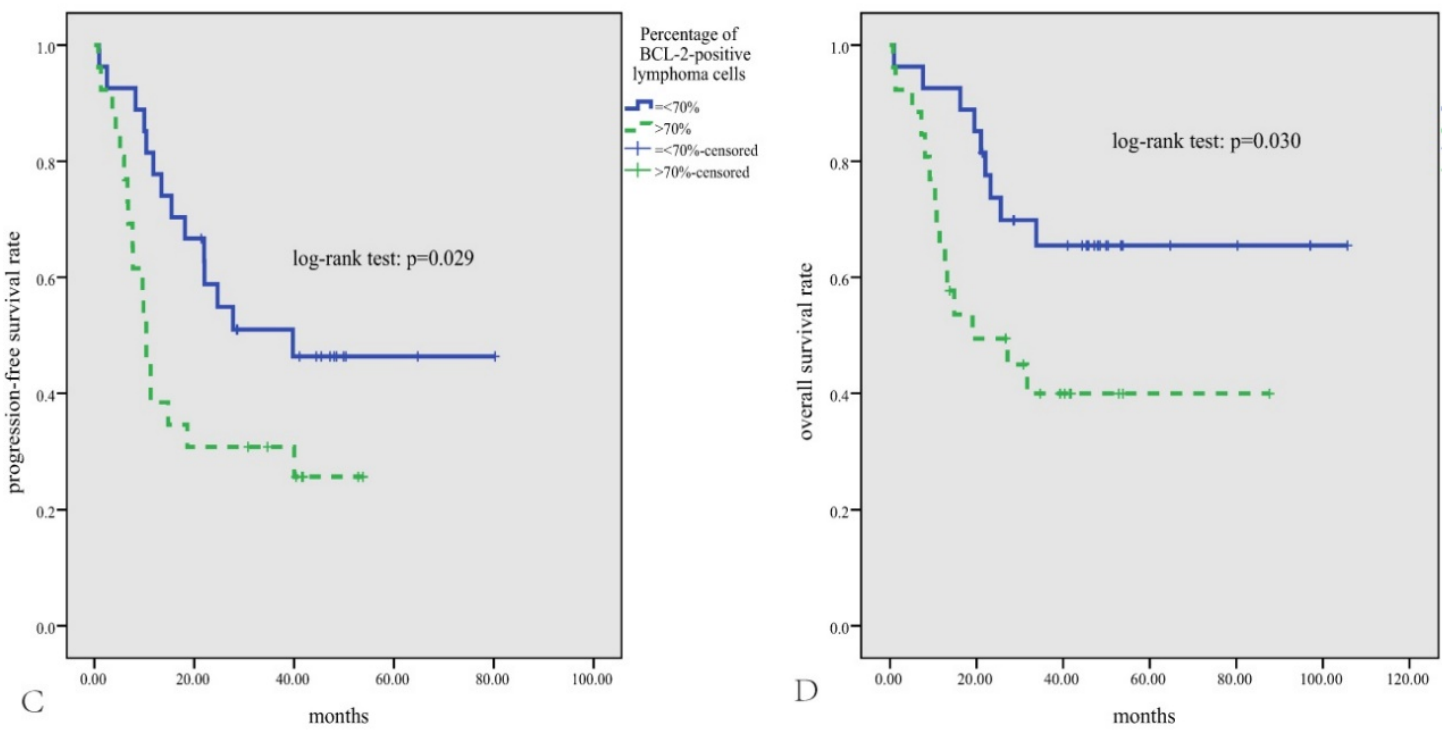

Percentage of BCL-2-positive mphoma cells

$\boldsymbol{r}=<70 \%$

$+=>70 \%$-censored $+>70 \%$-censored
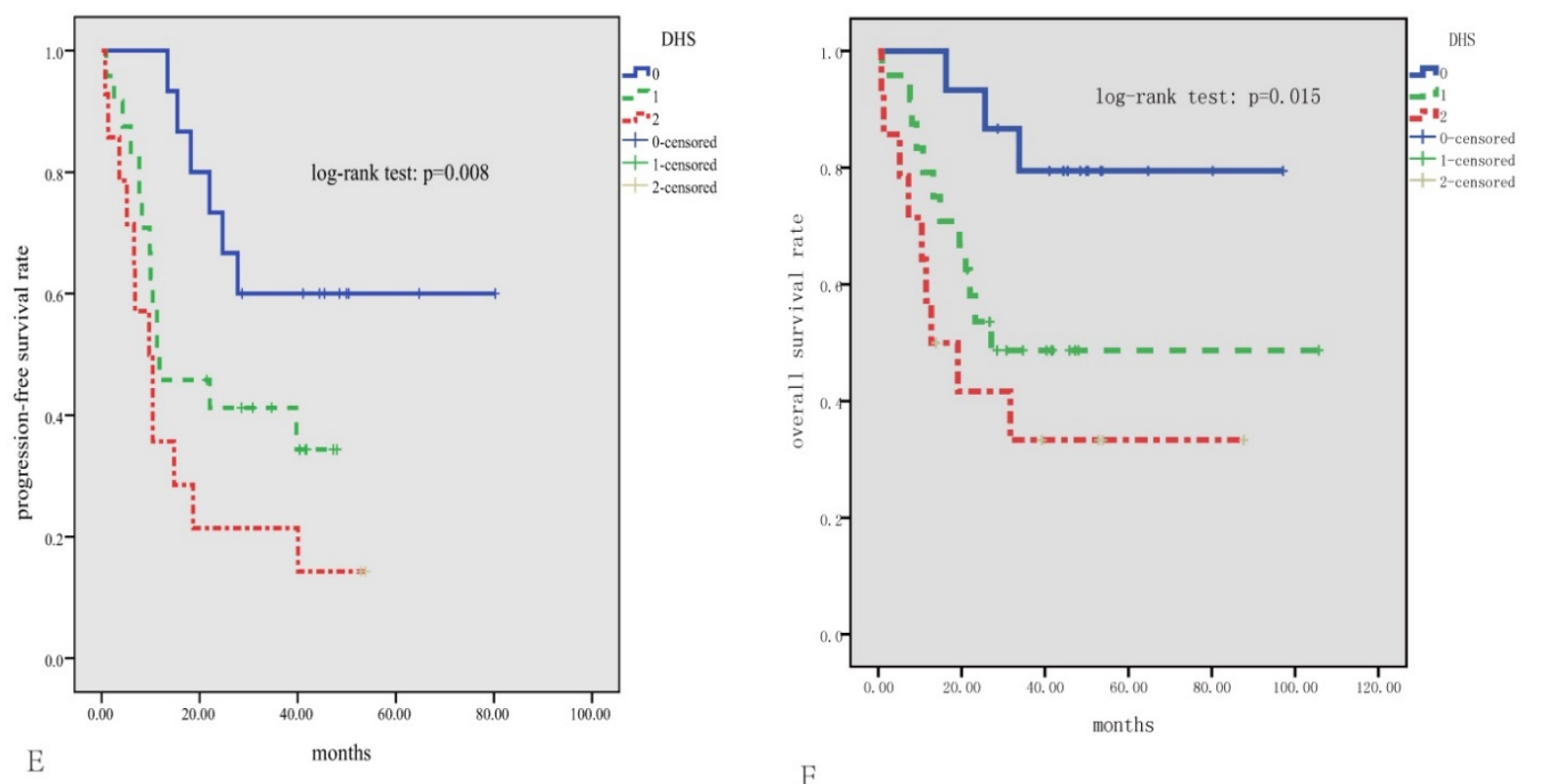

Figure 2. Survival analysis in the whole cohort of 53 patients with ENKTL. Patients with positive expression percentage $>20 \%$ for MYC (A, B) or $>70 \%$ for BCL2 (C, D) have significantly inferior PFS and OS; According to the DHS, patients could be divided into three significantly different risk groups for PFS and OS (E, F). 

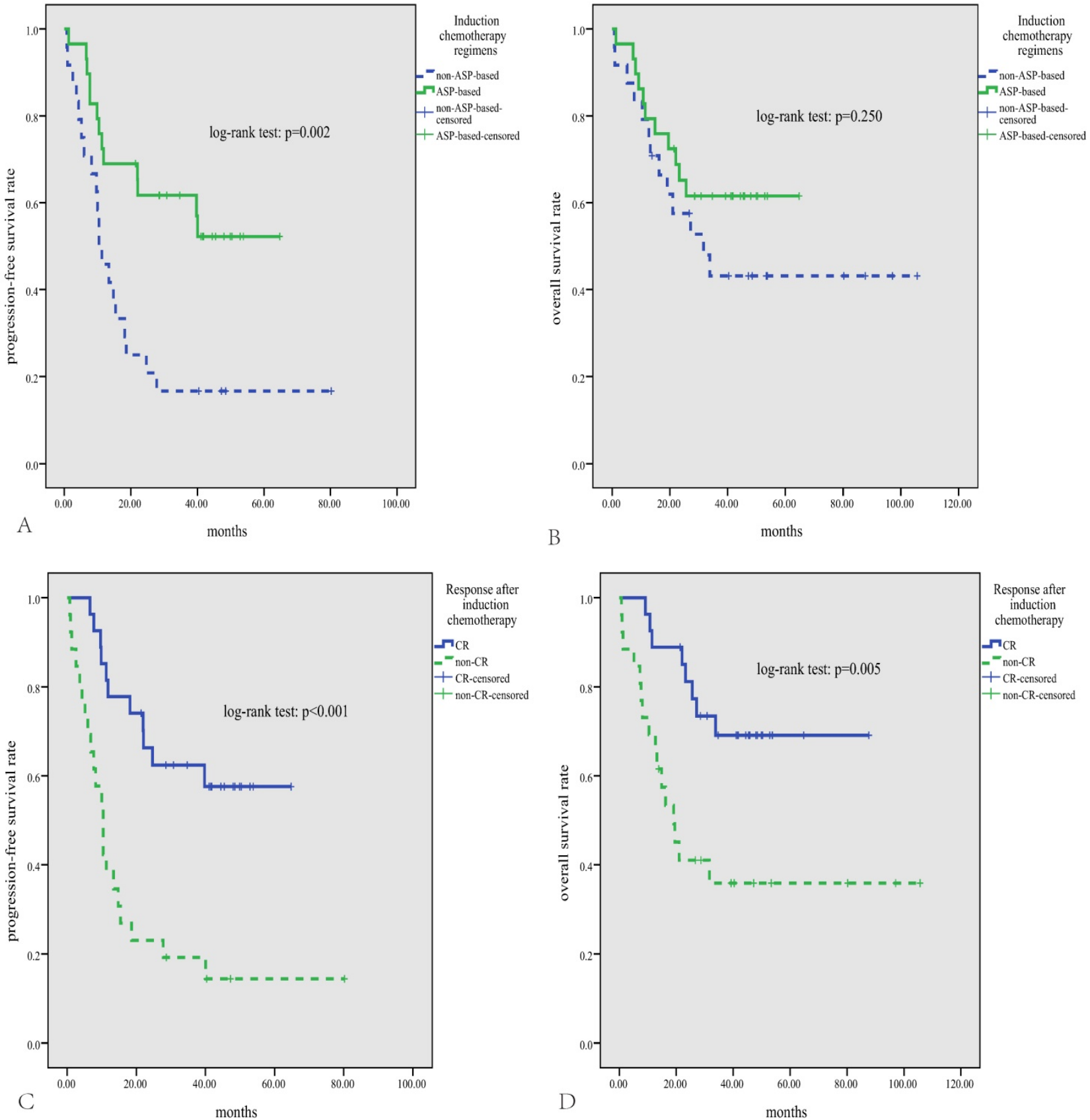

Figure 3. Survival analysis according to chemotherapy regimens and treatment response. Patients treated with asparaginase-based regimens have significantly superior PFS (A), and a trend for better OS (B); patients who get CR after treatment have significantly better PFS and OS (C, D).

\section{Discussion}

ENKTL is a rare neoplasm with heterogeneous clinical behaviors and survival outcomes. Although the IPI, and KPI have been shown to be useful clinical tools to risk stratify patients with ENKTL[8,10], they do not capture the biologic spectrum of this disease. NK/T lymphoma cells are almost invariably infected with EBV[17]. Analysis of the EBV terminal repeat region shows the presence of a clonal episomal form, implying EBV infection is etiologically associated with ENKTL[18]. EBV infection is mainly characterized by the expression of latent genes including EBNA1, LMP-1, LMP-2, and EBER[19]. Among them, LMP-1 has been identified as the main transforming oncoprotein of EBV, which can cause oncogenic transformation of EBV-infected cells[20]. Many researches suggest that LMP1 is frequently expressed in a variety of EBV-associated cancers, including nasopharyngeal carcinoma (NPC), Hodgkin disease (HD), and ENKTL, which commonly signifies a poor prognosis[21,22]. As yet, it was known that LMP-1 can exert anti-apoptotic activity and promote proliferation by up-regulation of the expression of 

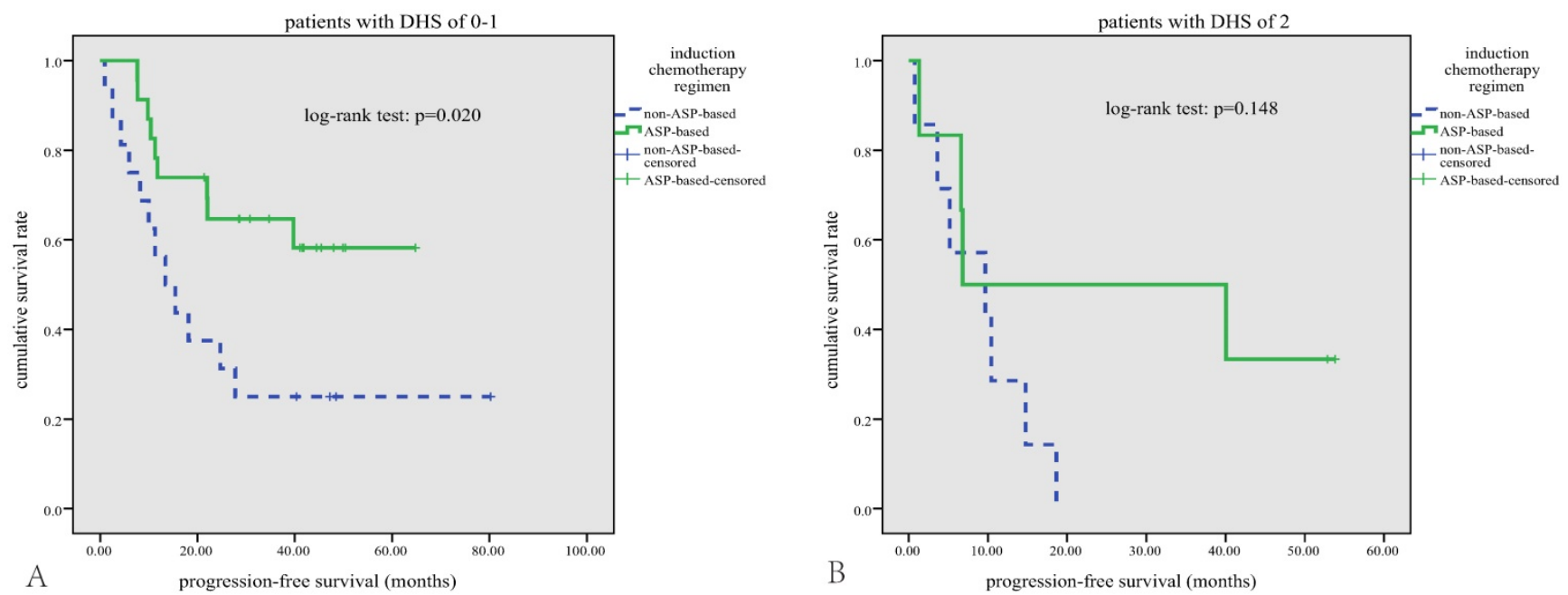

Figure 4. Subgroup survival analysis. In patients with DHS of $0-1$, those who received asparaginase-based regimens have better PFS (A); this survival benefit of asparaginased-based regimens was diminished in patients with DHS of 2 (B).

BCL2 and MYC[23].BCL2 is a central anti-apoptotic gene, and the overexpression has been found to be a marker of poor prognosis in several studies[24].The MYC oncogene can encode a transcription factor that promotes cell growth and proliferation. Previous study demonstrated that MYC staining was detected in a high proportion of T-cell (94\%) and NK-cell (95\%) lymphomas, with the highest expression detected in T-lymphoblastic lymphoma and ENKTL[25]. Furthermore, research shows that MYC protein expression correlate with inferior survival in patients with ENKTL[26].

In this study, we performed combined detection of BCL2 and MYC protein expression by immunohistochemistry method to investigate the clinical correlations in newly diagnosed ENKTL. We found that either high expression of MYC or BCL2 was significantly correlated with inferior PFS and OS, which is consistent with that previously reported $[26,24,15]$. When we applied the DHS to our 53 patients, it was able to identify three categories of patients with significantly different prognosis. The high expression of both MYC and BCL2-the patients with a DHS of 2-indicates the worst prognosis of ENKTL. Conversely, the patients with a DHS of 0 had better outcomes. Furthermore, we proved that DHS was an independent prognostic factor for both PFS and OS. Notably, the DHS was not associated with patients' clinical characteristics including age, gender, disease stage, LDH level, B symptoms, performance status, or local tumor invasiveness, some of which were significant prognostic parameters in IPI, and KPI[10]. Thus we think the DHS can supplement risk stratification for newly diagnosed ENKTL from the viewpoints of etiology. In addition, no MYC or BCL2 rearrangement was observed on 5 tissue sections with DHS of 2 through FISH analysis, so other oncogenic mechanisms beside MYC or BCL2 translocation are likely to be involved in upregulation of MYC and BCL2 protein expression in ENKTL[15]. As an EBV-associated lymphoma, ENKTL overexpress LMP-1[16]. Using NK-92 cell line (weak expression of LMP-1), we demonstrated increased expression of both MYC and BCL2 after transfection of LMP-1 into NK-92 cell line (Supplement Figure 1), which supported our hypothesis that EBV infection could induce expression of MYC and BCL2 independent of gene translocations. We hold the view that proteins are the functional molecules that are derived from gene activity, and protein expression levels represent a more direct measure of the activity of a particular gene, so the immunohistochemical DHS can better evaluate prognosis for newly diagnosed ENKTL than genetic methods, mainly FISH.

In terms of treatment modalities, a general improvement in survival rates has been achieved since asparaginase-based CT regimens were used to treat this disease[5,7]. The anticancer effect of asparaginase is not affected by MDR due to its unique mechanism: ENKTL cells unable to synthesize asparagine die when their stores of asparagine are depleted by asparaginase[27]. In our study, 54.7\% patients treated with asparaginase-based CT regimens showed a better response rate and a better PFS compared with those treated with anthracycline-based CT regimens, which further proved the value of asparaginase in treatment of ENKTL. No significant difference in OS between the treatment groups was noted, probably due to the small sample size and short follow-up period. In subgroup analysis, asparaginase-based CT can significantly improve the survival outcomes in patients with DHS of 0-1(Figure 4-A). However, this 
survival benefit of asparaginase-based CT was diminished in patients with DHS of 2 (Figure 4-B), implicating that MYC and BCL-2 expression may mediated the resistance mechanisms to asparaginase.

In conclusion, our study demonstrates that DHS can help identify patients with newly diagnosed ENKTL who are at a high risk for a poor clinical outcome. It is crucial to early and accurately identify high-risk patients in order to improve survival in this subset of lymphoma patients.

\section{Supplementary Material}

Supplementary figure 1.

http://www.jcancer.org/v08p0793s1.pdf

\section{Acknowledgements}

This work was supported by the National Natural Science Foundation of China [contract/grant number: 81400159]; Outstanding Young Talents Project of Sun Yat-sen University Cancer Center [contract/grant number:04190101\#]; and Clinical Medical Scientist project of Sun-Yat sen University Cancer Center [contract/grant number:09020101\#]. We would like to thank all of the treating physicians of Sun Yat-sen University Cancer center for allowing us to include their patients.

\section{Abbreviations}

ENKTL: Extranodal NK/T-cell lymphoma, nasal type; RT: radiotherapy; CT: chemotherapy; MDR: multi-drug resistance; IPI: international prognostic index; KPI: Korean Prognostic Index; LDH: lactate dehydrogenase; PINK: prognostic index for natural killer cell lymphoma; LMP-1: latent membrane protein-1; DHL: double-hit lymphoma; DHS: double-hit score; IFRT: involved-field radiotherapy; IMRT: intensity modulated radiotherapy; ECOG: Eastern Cooperative Oncology Group; PS: performance status; LTI: local tumor invasion; ASP: asparaginase; CR: complete response.

\section{Competing Interests}

The authors have declared that no competing interest exists.

\section{References}

[1] $\mathrm{Au} W Y$, Weisenburger DD, Intragumtornchai $\mathrm{T}$, et al. Clinical differences between nasal and extranasal natural killer/T-cell lymphoma: a study of 136 cases from the International Peripheral T-Cell Lymphoma Project. Blood. 2009;113 (17):3931-3937.

[2] Yoon TY, Lee HT, Chang SH. Nasal-type T/natural killer cell angiocentric lymphoma, Epstein-Barr virus-associated, and showing clonal T-cell receptor gamma gene rearrangement. The British journal of dermatology. 1999;140 (3):505-508

[3] Li YX, Wang H, Jin J, et al. Radiotherapy alone with curative intent in patients with stage I extranodal nasal-type NK/T-cell lymphoma. International journal of radiation oncology, biology, physics. 2012;82 (5):1809-1815.
[4] Wang B, Li XQ, Ma X, et al. Immunohistochemical expression and clinical significance of P-glycoprotein in previously untreated extranodal NK/T-cell lymphoma, nasal type. American journal of hematology. 2008;83 (10):795-799.

[5] Wang L, Wang ZH, Chen XQ, et al. First-line combination of gemcitabine, oxaliplatin, and L-asparaginase (GELOX) followed by involved-field radiation therapy for patients with stage IE/IIE extranodal natural killer/T-cell lymphoma. Cancer. 2013;119 (2):348-355.

[6] Lin N, Song Y, Zheng W, et al. A prospective phase II study of L-asparaginaseCHOP plus radiation in newly diagnosed extranodal NK/T-cell lymphoma, nasal type. Journal of hematology \& oncology. 2013;6:44.

[7] Jaccard A, Gachard N, Marin B, et al. Efficacy of L-asparaginase with methotrexate and dexamethasone (AspaMetDex regimen) in patients with refractory or relapsing extranodal NK/T-cell lymphoma, a phase 2 study. Blood. 2011;117 (6):1834-1839.

[8] Wang L, Xia ZJ, Huang HQ, et al. Cyclophosphamide, doxorubicin, vincristine, and prednisone (CHOP) in the treatment of stage IE/IIE extranodal natural killer/T cell lymphoma, nasal type: 13-year follow-up in 135 patients. International journal of hematology 2012. 96 (5):617-623.

[9] Wang L, Xia ZJ, Lu Y, et al. A modified international prognostic index including pretreatment hemoglobin level for early stage extranodal natural killer/T cell lymphoma. Leukemia \& lymphoma. 2015;1-7.

[10] Lee J, Suh C, Park YH, et al. Extranodal natural killer T-cell lymphoma, nasal-type: a prognostic model from a retrospective multicenter study. Journal of clinical oncology : official journal of the American Society of Clinical Oncology. 2006;24 (4):612-618.

[11] Kim SJ, Yoon DH, Jaccard A, et al. A prognostic index for natural killer cell lymphoma after non-anthracycline-based treatment: a multicentre, retrospective analysis. The Lancet Oncology. 2016;17 (3):389-400.

[12] Friedberg JW. Double-hit diffuse large B-cell lymphoma. Journal of clinical oncology : official journal of the American Society of Clinical Oncology. 2012;30 (28):3439-3443.

[13] Cohen JB, Geyer SM, Lozanski G, et al. Complete response to induction therapy in patients with Myc-positive and double-hit non-Hodgkin lymphoma is associated with prolonged progression-free survival. Cancer. 2014;120 (11):1677-1685.

[14] Petrich AM, Gandhi M, Jovanovic B, et al. Impact of induction regimen and stem cell transplantation on outcomes in double-hit lymphoma: a multicenter retrospective analysis. Blood. 2014;124 (15):2354-2361.

[15] Green TM, Young KH, Visco C, et al. Immunohistochemical double-hit score is a strong predictor of outcome in patients with diffuse large B-cell lymphoma treated with rituximab plus cyclophosphamide, doxorubicin, vincristine, and prednisone. Journal of clinical oncology : official journal of the American Society of Clinical Oncology. 2012;30 (28):3460-3467.

[16] Wang ZY, Liu QF, Wang H, et al. Clinical implications of plasma Epstein-Barr virus DNA in early-stage extranodal nasal-type NK/T-cell lymphoma patients receiving primary radiotherapy. Blood. 2012;120 (10):2003-2010.

[17] Kwong YL. Natural killer-cell malignancies: diagnosis and treatment. Leukemia. 2005;19 (12):2186-2194

[18] Siu LL, Chan JK, Kwong YL. Natural killer cell malignancies: clinicopathologic and molecular features. Histology and histopathology. 2002;17 (2):539-554.

[19] Izumi KM. Identification of EBV transforming genes by recombinant EBV technology. Seminars in cancer biology. 2001;11 (6):407-414.

[20] Kobayashi H, Nagato T, Takahara M, et al. Induction of EBV-latent membrane protein 1-specific MHC class II-restricted T-cell responses against natural killer lymphoma cells. Cancer research. 2008;68 (3):901-908.

[21] Herbst H, Dallenbach F, Hummel M, et al. Epstein-Barr virus latent membrane protein expression in Hodgkin and Reed-Sternberg cells. Proceedings of the National Academy of Sciences of the United States of America. 1991;88 (11):4766-4770.

[22] Fahraeus R, Fu HL, Ernberg I, et al. Expression of Epstein-Barr virus-encoded proteins in nasopharyngeal carcinoma. International journal of cancer. 1988;42 (3):329-338.

[23] Henderson S, Rowe M, Gregory C, et al. Induction of bcl-2 expression by Epstein-Barr virus latent membrane protein 1 protects infected B cells from programmed cell death. Cell. 1991;65 (7):1107-1115.

[24] Barrans SL, Evans PA, O'Connor SJ, et al. The $t(14 ; 18)$ is associated with germinal center-derived diffuse large B-cell lymphoma and is a strong predictor of outcome. Clinical cancer research : an official journal of the American Association for Cancer Research. 2003;9 (6):2133-2139.

[25] Chisholm KM, Bangs CD, Bacchi CE, et al. Expression profiles of MYC protein and MYC gene rearrangement in lymphomas. The American journal of surgical pathology. 2015;39 (3):294-303.

[26] Huang X, Sun Q, Fu H, et al. Both c-Myc and Ki-67 expression are predictive markers in patients with extranodal NK/T-cell lymphoma, nasal type: a retrospective study in China. Pathology, research and practice. 2014;210 (6):351-356.

[27] Obama K, Tara M, Niina K. L-asparaginase-Based induction therapy for advanced extranodal NK/T-cell lymphoma. International journal of hematology. 2003;78 (3):248-250. 